\title{
Adaptive Continuous Mutation Model Genetic Algorithm
}

\author{
M. G. Can, B. B. Oner, H. Kurt
}

\begin{abstract}
-
Genetic algorithm (GA) is a stochastic optimization approach modelling the principles of natural evolution. Since its first proposal in the literature almost six decades ago, various optimization and search problems have been tackled by means of either basic version of GA or its modified forms. In this study, some certain modifications were implemented on initial population, selection, crossover and mutation phases of GA's main flowchart. The mutation process has been described as individual based adaptive mutation that varies continuously for the first time in the literature and the performance of the proposed GA (PGA) with the all modifications was compared with the standard GA (SGA) for two different test problems. As a result, it was seen that PGA could approach the optimum point with more than 100 times better result within 30 times shorter run-time compared to the SGA.
\end{abstract}

Index Terms - Evolutionary computation, fast evolutionary algorithms, genetic algorithm, global optimization, heuristic optimization

\section{INTRODUCTION}

$\mathrm{T}$ HE genetic algorithm (GA) is a method to solve optimization problems based on a natural selection process that mimics biological evolution. This model, which was developed by analogy with the survival feature of the living being who adapts best to the conditions of nature, has been used in many different optimization problems in the literature such as computational science, lens design, operational researches [1-5]. The distinct superiority of GA is its nature to find a global optimum via performing a global search on each iteration while it is converging on a specific solution. This makes GA extremely convenient to be used on optimization problems including multi-variable, nonlinear and multiple disconnected feasible regions. Even so, it also has some downsides besides its well-known advantages. Solution space of a conventional genetic algorithm is constituted by the genes of the first generations. In other words, the upper limit of the optimal solution is limited by the genetic structure of the first generation, even the overall performance increases proportionally by the number of the generations. For instance, GA is quite ideal to implement on problems in which

Manuscript received June 6, 2021

M. G. Can is with the Electrical and Electronics Engineering Department, TOBB University of Economics and Technology, Sogutozu, Ankara, 06560 Turkey, on leave from Roketsan Missile Inc., Ankara, Turkey (e-mail: m.can@etu.edu.tr) individuals are represented as binary strings. On the other hand, GA performance is strictly limited for the search and optimization problems where the solution space is constituted from real numbers. Even if a longer bit array enables a better sampling, it also causes a computationally harder solution process. Mutation operand adds some variation to genes and tries to step over the borders of initially generated genetic structure. However, this operand brings a limited contribution to the success of GA due to its totally randomized nature of implementation.

In this study, the aforementioned issues of standard genetic algorithm (SGA) is addressed by inserting a controlled and continuous mutation operand to the algorithm. In the proposed genetic algorithm (PGA), mutation is not the random variation of a selected binary string but a treatment for every gene with additional Gaussian noise whose variance is determined with the success of the individual. Chromosomes of two individuals are exposed to a controlled mutation during the crossover stage. This scheme sets the variance of the mutation to be inversely proportional to the success of parent individuals. Thus, genes of successful individuals are exposed a local variation. On the contrary, chromosomes of less successful parent individuals are exposed to mutations with larger variances. This enables a quick convergence to the optimum solution by keeping the successful chromosome structure of a previous generation while changing the unsuccessful ones.

In the next section, the comparison of the SGA and PGA is explained in detail such that the required modifications on SGA is explained. This comparison is enhanced on the Discussion section by implementing the proposed algorithm on two different problems and the corresponding results present the performance improvement over SGA. The article is concluded by final comments and possible further improvements on the current approach.

\section{METHOD}

GA is well-known by the evolutionary algorithm community. Therefore in this subsection the possible modifications on the SGA phases to improve rapidity and performance are briefly described. At the end of each phase, the parts where and why

B.B. Oner is with the Physics Department, Gazi University, Yenimahalle, Ankara, 06560 Turkey, on leave from Roketsan Missile Inc., Ankara, Turkey, on leave from Roketsan Missile Inc., Ankara, Turkey

H. Kurt, is with the School of Electrical Engineering, KAIST, Daejeon, Republic of Korea (e-mail: hamzakurt@kaist.ac.kr). 


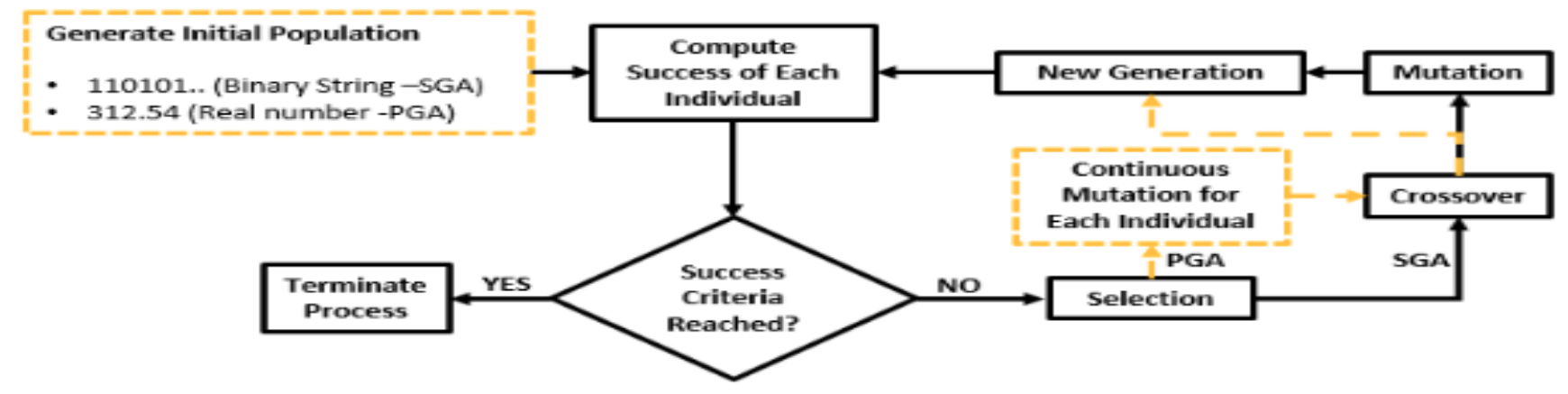

PGA: Proposed GA with Continuous Controlled Mutation Operand

SGA: Standard GA

Fig. 1. Standard Genetic Algorithm Flow Chart in which proposed modifications on the algorithm are shown within the dashed boxes.

the distinctions of the PGA will come out are pointed out. In Figure 1 SGA flowchart and proposed modifications on this chart is given.

\section{A. Initial Population}

SGA is a method where multiple, independent and random initial solutions are needed in this phase. Conventionally, genes are defined with a bit array. Longer arrays naturally requires much larger population sizes to obtain an efficient optimization because of the variety of extended genetic permutations. This trade-off manifests itself especially on "global extremum" problems. A major modification can be implemented addressing this issue by suppressing the algorithm run time as well as increasing the resolution of the solution space. As seen in Figure 1- Initial Population inset the defined solution space is continuous rather than discrete vectors corresponding to finite length bit arrays. In this manner, the definition of a gene is evolved to a real number within a specified interval. Thus, chromosomes denote real number arrays from now on. For example, assume that our problem is finding an array of 10 coefficients, $x_{N}$, where the sub-index $N$ takes values from 0 to 9. All coefficients are real numbers and it is known that they stay within the open interval of $[0,1023]$. The upper and lower limits of the solution space intervals will be denoted by $L_{u}$ and $L_{l}$, respectively. A reasonable solution suggestion is an individual with ten chromosomes, $S_{i}=\left(a_{i}^{n} ; n=0, \ldots, 9\right)$, where $S_{i}$ denotes the $i^{\text {th }}$ individual while $a_{i}^{n}$ denotes $n^{\text {th }}$ chromosome of $S_{i}$. A crossover between $i^{\text {th }}$ and $j^{\text {th }}$ individuals gives rise to a new individual with chromosomes which consist of some gene bit array parts of both $a_{i}^{n}$ and $a_{j}^{n}$.

Even if each chromosome is constituted by 10 digits, they may scan only the integers within the interval given in the aforementioned example when the conventional binary array approach is implemented:

Modification 1: Each chromosome should be defined as a real number but not quantized bit arrays.

A continuous solution space enables to select any value for each gene. Therefore, the modification to overcome the limited genetic permutation issue is proposed. However, this modification just provides a continuous move in solution space but not enough to lead to exact solution. So, authors decided to add another modification to manipulate every individual to migrate to the proximity of the exact solution which occurs in the mutation before the crossover phase.

\section{B. Success Computation}

A normalized fitness function of $f=1 /(1+g(\boldsymbol{r}))$ is commonly used in the literature [6] where $g(\boldsymbol{r})$ shows a performance criterion that may take values between $[0, \infty)$ and $\boldsymbol{r}$ has a dimension that corresponds to the number of genes per individual. The function $g$ is still fully-optional and depends on the specific problem. So, the choice of fit function is not only a PGA's but also any optimization algorithm's consideration. In the present study same success computation algorithm is utilized for both SGA and PGA.

\section{Selection}

Selection phase is also crucial in order to give a rise to a better generation. The most common two methods in the literature are Roulette-Wheel and Stochastic-Remainder Methods [6-9]. Each individuals' probability to transfer genes to the next generation should be determined regarding its normalized success. Again, these probability values are fully optional to choose and it is assumed that the reference SGA uses Roulette-Wheel method where the wheel is constituted by different size lots and the lot sizes are linearly proportional to the individuals' success.

Roulette-Wheel method allocates $f_{\mathrm{i}}$ fit values of each individual in a generation such that individuals with better fit values have more probability $\left(p_{\mathrm{RW}}\right)_{\mathrm{i}}$ to join the crossover phase. The method is conventional (linear) when these probability values are directly proportional with fit values, namely $\left(p_{R W}\right)_{i}=f_{i} / \sum_{j} f_{j}$ while in the most general case allocation formula can be written as:

$$
\text { Modification 2: }\left(p_{R W}\right)_{i}=\left(f_{i}\right)^{h} / \sum_{j}\left(f_{j}\right)^{h}
$$

where the summation is over all individuals in a single generation. In the proposed algorithm the final touch in this phase is on the parameter $h$. The more successful individual in one of the future generations should manifest itself clearly on allocation, since the individuals' fitness values' variance reduces by each generation. Therefore the $h$ treated as an exponential function of generation number $\left(\mathrm{n}_{\mathrm{g}}\right): h=\left(n_{g}\right)^{q}$ 

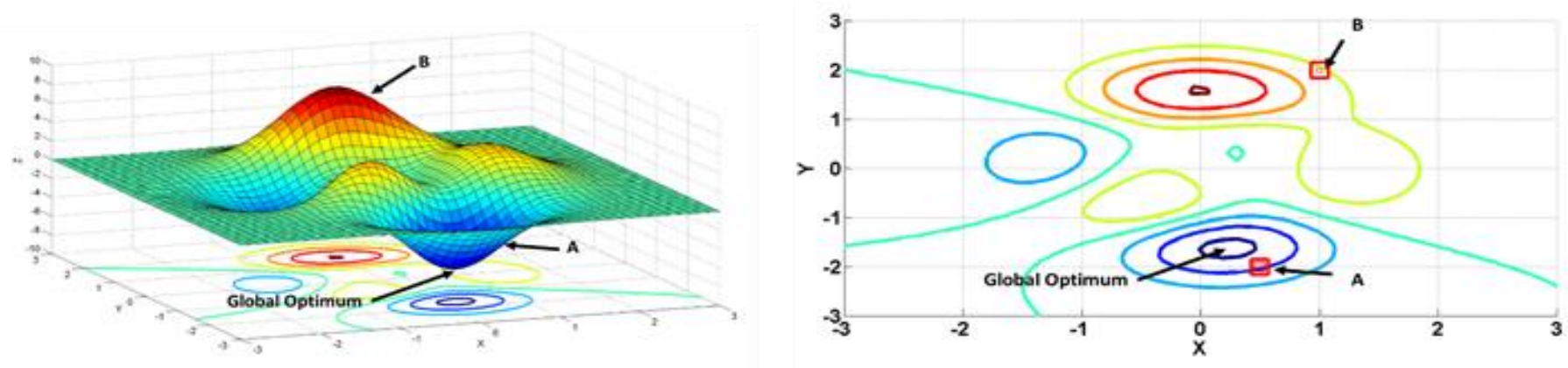

Fig. 2(a).Minimum finding problem where A and B randomly generated individuals. (b) Corresponding contour view of individuals and the global optimum where $q$ is constant.

\section{Crossover}

$1 / 2$ probability for a new individual is given to have a specific gene bit array to be taken from one of the parents in SGA. In the most general case, this probability is also another parameter such that an individual with a better fit function result may have more probability to transfer genes to the next generation. In other words, it may be a function of parent individuals' success. In a similar manner, a dominance parameter is defined between parents on crossover phase:

$$
\text { Modification 3: }\left(D_{R W}\right)_{i}=\left(f_{i}\right)^{h} / \sum_{j=1}^{2}\left(f_{j}\right)^{h}
$$

where the summation is over only parents.

\section{E. Mutation}

Crossover stage is necessary whereas mutation stage is optional but quite useful especially to increase the diversity of the population. In the present study this phase is proposed to be implemented on the parent individual genes before Crossover. The most critical parameter of this phase is the mutation probability $p_{m}$ of each gene. Even though, a constant probability with an optimal value (usually $p_{m} \ll 1$ ) is used in SGA, the proposed algorithm adds continuous mutation for each individual as it is seen in the Figure 1. Here at this point, a data link between mutation and success computation phases is proposed, in other words a controlled mutation. In the literature mutation phase is linked with the fitness function before [10]. However in this work individuals are binary strings and mutation is exposed to least significant bits or more significant bits relative to individuals' fitness value. In the present work, this data link manages continuous variation for all crossover and enables the algorithm to satisfy two constraints with added Gaussian noise:

i) The search should focus on the proximity of the current individual's chromosomes if that individual is estimated to be close to the optimum solution.

ii) The search should diverge from the proximity of current individual's chromosomes if that individual is estimated to be far from the optimum solution.

These constraints bring the last modification which makes the algorithm distinctive among the others:
Modification 4: All chromosomes should be exposed to mutation in such a way that the effect of the mutation should be inversely proportional to the success of the corresponding individual.

In this regard a mutation operand, $p_{m}$, as a Gaussian distributed noise centered on the gene with a variance as a function of the individual's success is proposed:

$$
\begin{aligned}
\left(p_{m}\right)_{i} & =\frac{1}{\sqrt{2 \pi} \sigma_{i}^{n}} e^{\sum_{i=1}^{k} \frac{\left(a_{i}^{n}\right)^{2}}{2\left(\sigma_{i}^{n}\right)^{2}}} \\
\sigma_{i}^{n} & =N_{n} e^{\left(f_{A} / f_{i}\right)}
\end{aligned}
$$

where $f_{A}$ is the average success of the population while $f_{i}$ is the fitness function of the individual and $k$ is the total number of genes of one individual. $\sigma_{\text {in }}$ denotes the variance of the mutation probability for $i^{\text {th }}$ individual's $n^{\text {th }}$ gene. Note that the variance is inversely proportional to $f_{i}$. Coefficient $N$ with the exponential term is to extend the variance and plays a remarkable role on the performance of the algorithm. A large choice may ruin the fit function of successful parents while a small choice reduces the efficiency of the method. $N$ is chosen such that $\pm 3 \sigma$ width of an exactly average successful individual to correspond $10 \%$ of the total interval.

Function 'peaks' are plotted as an example in Figure 2. The objective function is defined to find the minimum. Point ' $\mathrm{A}$ ' and point ' $\mathrm{B}$ ' are randomly selected as given in Figure 2. As can be seen clearly, the fitness function of the individual $\mathrm{A}$ is higher than the $\mathrm{B}$. Therefore, individual $\mathrm{A}$ is exposed to a normal distributed mutation with a narrower variance which forces it to variate in a limited region around point $\mathrm{A}$ before crossover. On the other hand individual B is treated with a mutation with wider variance. Illustrations of the mutation

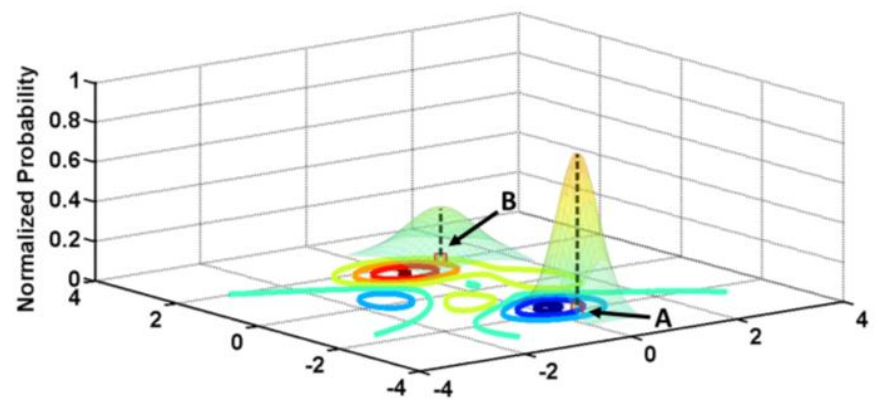

Fig. 3. Mutation probability distributions $\left(\mathrm{p}_{\mathrm{m}}\right)$ for two individuals. 
probability densities are given in Figure 3. As highlighted in Figure 1, this probabilistic continuous mutation is firstly proposed within the present paper and enables to enhance SGA performance remarkably with run-time and optimum search manner. The effect of this modification is analyzed in the Discussion section, which is dedicated to a comparison of PGA.

\section{DISCUSSION}

In this section enhancement of PGA over SGA is numerically demonstrated. The PGA approach has been implemented into two different problems. The first one is the above mentioned 10 different real number search problem within a specific interval. Each algorithm (SGA and PGA) has been run 1000 times for 20 generations (iterations) and initiated with 100 individuals. The arithmetic mean of the best individual's fitness of each run, $<$ $f_{\text {best }}>=<1 /\left(1+g_{\min }\right)>$, was computed for comparison. A normalized least squares method of the selection formula given below was used to calculate success for both algorithms:

$$
g_{i}=\sum_{n=1}^{10}\left(\left|a_{i n}-x_{n}\right| / E_{\text {max }}\right)^{2}
$$

where $E_{\max }$ denotes the possible maximum error. Obtained PGA and SGA results for real number search problem are given in the following Table 1. Global optimization toolbox of Mathworks [11] including genetic algorithm with mutation was used to make a fair comparison. Mutation variance of the SGA algorithm is given as

$$
\sigma_{n}=\left(L_{u}-L_{l}\right)\left[1-s \frac{N_{\text {ins }}}{N_{\max }}\right]
$$

where $s \leq 1$ is a constant, $N_{\text {ins }}$ and $N_{\max }$ are the instant and maximum number of generations, respectively. Note that the adaptive mutation variance $\sigma_{n}$ depends on the generation not the fitness of individual itself as hereby it's proposed for the first time in the literature.

As it is seen from the Table 1, bit array based SGA algorithm could only reach to the level of $\left\langle g_{\min }\right\rangle=6.0$ with initial population size 100 and within 20 iteration of generations. Meanwhile, PGA has achieved to increase the fitness value over $\left\langle f_{\text {best }}\right\rangle=99.9 \%$ with same population size within 20 iterations. PGA also performs 5 times better optimum search compared to the literature [10]. The capability of the proposed algorithm becomes clearer when the other algorithms were run for 100 generations. PGA still performs more than 2 times better within only 20 generations. Please see Figure 4 for to compare ability of SGA and PGA to approach the solution. PGA has an obvious superiority over SGA in terms of finding better solutions within a less run-time.

Even though each generation has a better average success after each iteration of SGA, modifications of PGA catalyze this improvement process dramatically.

Table 1 SGA and PGA performance comparison for 10 real number search. Population size (Number of individuals per generation) is hold fixed as 100 for all optimizations.

\begin{tabular}{cccc} 
Modifications & \# Generations & $\begin{array}{c}\text { Mean Run } \\
\text { Time (ms) }\end{array}$ & $\left\langle\mathbf{g}_{\min }\right\rangle$ \\
\hline \hline \multicolumn{5}{c}{ SGA } \\
\hline \hline \multicolumn{5}{c}{ Literature } \\
\hline 1 & 20 & 43.80 & 6.0 \\
\hline $1, p_{m}=3 \%$ & 100 & 137.4 & $2.8 \times 10^{-2}$ \\
\hline $1, s=1$ & 100 & 130.7 & $1.4 \times 10^{-3}$ \\
\hline \hline \multicolumn{5}{c}{ PGA } \\
\hline $1,2,3$ & 20 & 9.000 & $6.8 \times 10^{-3}$ \\
\hline $1,2,3,4$ & 20 & 8.728 & $7.6 \times 10^{-4}$ \\
\hline $1,2,3,4$ & 100 & 42.59 & $3.2 \times 10^{-4}$ \\
\hline
\end{tabular}

The second problem that has been addressed in order to compare the SGA and PGA is a global extremum problem with a benchmark function:

$$
R(x, y)=20+x^{2}+y^{2}-10 *[\cos (2 \pi x)+\cos (2 \pi y)]
$$

which is also known as Rastrigin function. Main property of Rastrigin function is its non-monothon structure which gives multiple minima and maxima within a specific interval. Here, this interval is chosen to be $(-5,5)$ as in the previous case. It is
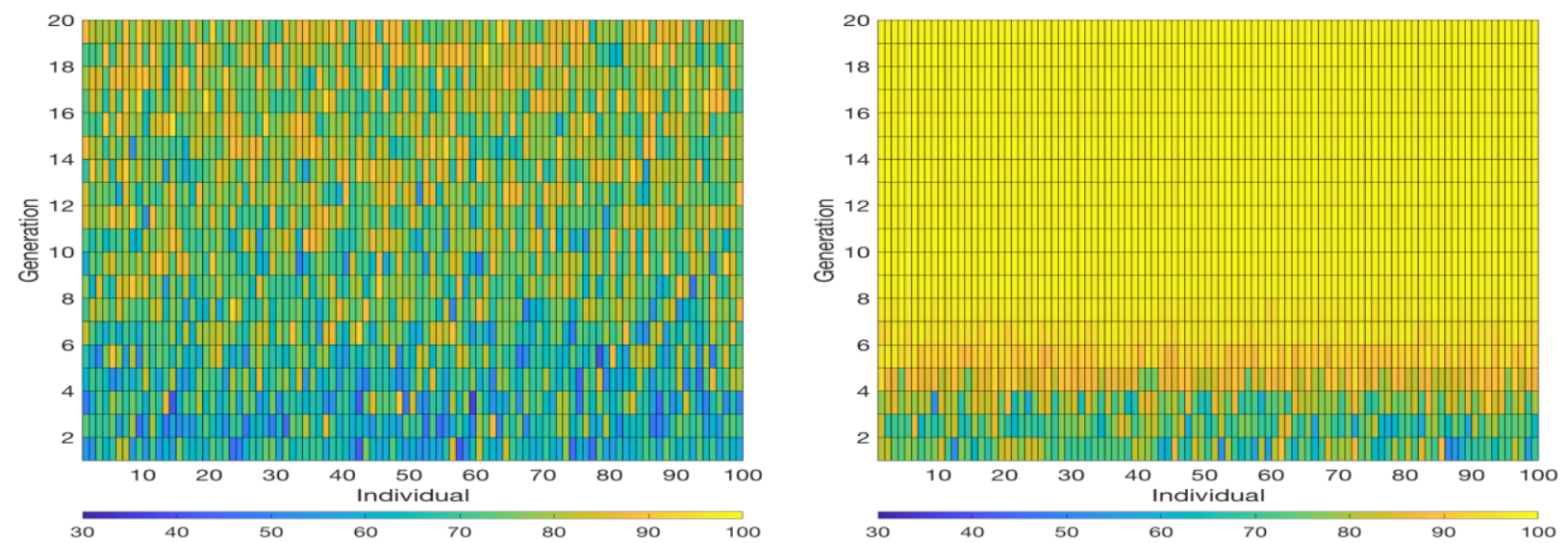

Fig. 4. Fitness of each individual is given for (a) SGA and (b) PGA. Colourbar shows the $f \%$ values and results were computed in a single-run. The fitness function is taken as $f_{i}=100\left(E_{\max }-E_{i}\right) / E_{\max }$ for this plot. 
assumed that coefficients of the problem are known but the ( $x$, $y$ ) position of global minimum is not. Note that, if the coefficients of the function were not known it also could be treated as in the first case. In Table 2, SGA and PGA results are given. SGA results are compared with the literature and expected success is verified [12]. As clearly seen from Table 2, with the PGA it is possible to have more than 40 times better result within 3 times less run time.

Table 2 SGA and PGA performance comparison for global minima search problem (Rastrigin).

\begin{tabular}{|c|c|c|c|}
\hline Modifications & \# Generations & $\begin{array}{l}\text { Mean Run } \\
\text { Time (ms) } \\
\end{array}$ & $\left\langle\mathrm{g}_{\min }\right\rangle$ \\
\hline \multicolumn{4}{|c|}{ SGA } \\
\hline \multirow{2}{*}{$1, p_{m}=3 \%$} & 20 & 41.89 & 1.53 \\
\hline & 100 & 77.83 & 1.35 \\
\hline \multicolumn{4}{|c|}{ PGA } \\
\hline \multirow{2}{*}{$1,2,3,4$} & 20 & 5.962 & 0.26 \\
\hline & 100 & 27.75 & 0.03 \\
\hline
\end{tabular}

\section{CONCLUSION}

As the conclusion, in the present work 4 different modifications are proposed to implement into the SGA. The first one is using real number instead of binary array to define the genes. Even if this modification is not proposed for the first time in the literature it is a crucial part of the study since it enables to implement the $4^{\text {th }}$ modification performed in the present study. The value of the most significant bit increases remarkably as gene bit array gets longer in the SGA and a totally random mutation affecting the most significant bit on such a chromosome may ruin a solution near to the optimum point by leading it to a random position on the solution space. This brings out the requirement for controlled mutation. As the $4^{\text {th }}$ modification, present study proposes an adaptive continuous mutation for every individual before crossover stage for the first time in the literature. This approach provides to keep successful individuals almost same while unsuccessful ones alter dramatically. Mutation is implemented on the continuous values as a normal distributed noise and variants are linked to the fitness values of the individuals. This scheme remarkably boosts the performance and run-time of the algorithm. The $2^{\text {nd }}$ and $3^{\text {rd }}$ modifications are implemented on selection and crossover phases, respectively. These modifications also has significant effect on the PGA's overall performance.

To sum up, heuristic models are very popular for large scale search problems and produce relatively good solutions with a guarantee of neighborhood to optimality. Likewise, GA is an awesome algorithm for the optimal search. However, it is not commonly utilized to solve non-polynomial (NP) problems such as optical design problems. The NP difficulty of the problems makes them difficult to realize the optimum solution in a reasonable time while looking for the global minimum. Thus, designer shall generate a new design starting from a hot start point based on previous designs and experiences. The PGA is promising algorithm to be utilized for the optimization of NP and NP-hard problems. Thus, it may enable to find global optimum of NP problems without any bias in the optimization process.

\section{REFERENCES}

[1] J. H. Holland, "Genetic algorithms.", Scientific American, vol. 267(1), pp. 66-73, 1992.

[2] D.E. Goldberg, "Genetic Algorithms", Optimization \& Machine Learning, 1989.

[3] E. S. Hou, N. Ansari, and H. Ren, "A genetic algorithm for multiprocessor scheduling," IEEE Transactions on Parallel and Distributed systems, vol. 5(2), pp. 113-120, 1994.

[4] K. Höschel and V. Lakshminarayanan, "Genetic algorithms for lens design: a review", Journal of Optics, vol. 48.1, pp.134-144, 2019

[5] P.C. Chu and J. E. Beasley, "A genetic algorithm for the multidimensional knapsack problem.", Journal of heuristics, vol. 4.1, pp. 63-86, 1998

[6] G. R. Harik, F. G. Lobo and D. E. Goldberg, "The compact genetic algorithm", IEEE transactions on evolutionary computation, vol.3, no 4, pp.287-297, 1994

[7] A. Thammano and W. Teekeng, "A modified genetic algorithm with fuzzy roulette wheel selection for job-shop scheduling problems", International Journal of General Systems, vol.44:4, pp.499-518,2015

[8] A. Lipowski, and D. Lipowska. "Roulette-wheel selection via stochastic acceptance." Physica A: Statistical Mechanics and its Applications, vol. 391.6, pp. 2193-2196, 2012.

[9] D. Whitley, "A genetic algorithm tutorial." Statistics and computing, vol. 4.2, pp. 65-85, 1994.

[10] S. M. Libelli, and P. Alba. "Adaptive mutation in genetic algorithms." Soft computing, vol. 4, no. 2, pp. 76-80, 2000

[11] A. Chipperfield et al., "Genetic algorithm toolbox for use with MATLAB.", 1994.

[12] P. Bajpai, and K. Manoj, "Genetic algorithm-an approach to solve global optimization problems." Indian Journal of computer science and engineering, vol.1.3, pp.199-206,

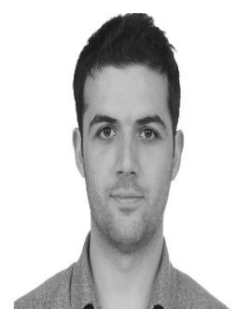

Melih G. Can was born in Samsun, TURKEY in 1989. He received the B.S. and M.S. degrees in Electrical and Electronics engineering from the TOBB University of Economics and Technology. He is currently pursuing the Ph.D. degree in electronics engineering at TOBB University, Ankara, TURKEY.

From 2012 to 2014, he was a Research Assistant with the Institute of Science, TOBB University. His research interest includes the nanoscale photonic structures, development of high cavity and sensing applications. He is now working in a designer position in the aerospace technology company Roketsan Missiles Inc., Ankara, TURKEY.

His research interest includes the nanoscale photonic structures, development of high cavity and sensing applications. surface processing and biological/medical treatment techniques using nonthermal atmospheric pressure plasmas, fundamental study of plasma sources, and fabrication of micro- or nanostructured surfaces. 


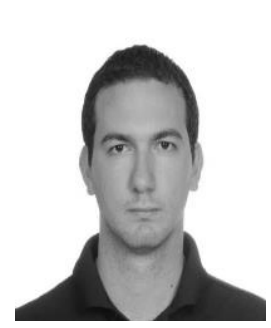

Bilgehan B. ÖNER was born in Ankara/TURKEY in 1991. He received the B.S. and M.S. degrees in Electrical and Electronics Engineering from TOBB University of Economics and Technology (TOBB UET) by full-scholarship. From 2013 to 2019, he was also Research Assistant in TOBB UET. He currently is a $\mathrm{Ph} . \mathrm{D}$. student of physics in Gazi University and quality engineer in aerospace technology company Roketsan Missiles Inc., Ankara, TURKEY. His past academic studies include 13 journal publications, 18 conference papers and review experiences. Author has a wide research interest from optical/photonics designs and optimizations to computational \& theoretical physics.

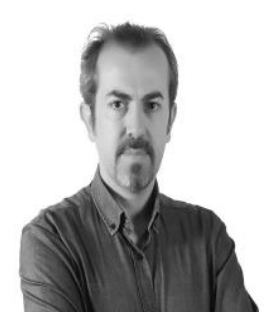

Prof. Hamza Kurt, received the B.S. degree from Middle East Technical University, Ankara in 2000, M.S. degree from the University of Southern California, Los Angeles in 2002, and Ph.D. degree from the Georgia Institute of Technology, Atlanta in 2006, all in electrical and electronics engineering. He was a research fellow with the Cedars-Sinai Medical Center, Biophotonics Research Lab, Los Angeles, from 2001 to 2002. He was a postdoctoral research Fellow with Georgia Tech for a short period of time and spent a year with the Institut d'Optique Graduate School, Paris as a postdoctoral scientist. He joined TOBB University in 2008 and stayed there until 2020. Prof. Kurt has joined the School of Electrical Engineering at KAIST in September 2020. He has published 126 peer-reviewed scientific papers including two invited papers and six single-author publications. He has given contributed and invited presentations and published more than 120 conference abstracts and proceedings. His current research interests include nanophotonics including the design and analysis of nano-photonic materials and structures for the realization of wavelength and sub-wavelength scale optical elements, metasurfaces, inverse design, slow light structures, graded-index optics, topological photonic insulators, solar energy, and optical biosensors. Prof. Kurt is a member of the Turkish Academy of Sciences and director of the MetaPhotonics Research Lab at KAIST. 